\title{
An Unsupervised Approach to Inferring the Localness of People Using Incomplete Geotemporal Online Check-In Data
}

\author{
CHAO HUANG, DONG WANG, and JUN TAO, University of Notre Dame
}

\begin{abstract}
Inferring the localness of people is to classify people who are local residents in a city from people who visit the city by analyzing online check-in points that are contributed by online users. This information is critical for the urban planning, user profiling, and localized recommendation systems. Supervised learning approaches have been developed to infer the location of people in a city by assuming the availability of high-quality training datasets with complete geotemporal information. In this article, we develop an unsupervised model to accurately identify local people in a city by using the incomplete online check-in data that are publicly available. In particular, we develop an incomplete geotemporal expectation maximization (IGT-EM) scheme, which incorporates a set of hidden variables to represent the localness of people and a set of estimation parameters to represent the likelihood of venues to attract local and nonlocal people, respectively. Our solution can accurately classify local people from nonlocal nones without requiring any training data. We also implement a parallel IGT-EM algorithm by leveraging the computing power of a graphic processing unit (GPU) that consists of 2,496 cores. In the evaluation, we compare our new approach with the existing solutions through four real-world case studies using data from the New York City, Chicago, Boston, and Washington, DC. The results show that our approach can identify the local people and significantly outperform the compared baselines in estimation accuracy and execution time.
\end{abstract}

CCS Concepts: • Computer systems organization $\rightarrow$ Embedded systems; Redundancy; Robotics; $\bullet$ Networks $\rightarrow$ Network reliability;

Additional Key Words and Phrases: Localness of people, unsupervised learning, online social networks, crowdsourcing, maximum likelihood estimation, GPU implementation

\section{ACM Reference Format:}

Chao Huang, Dong Wang, and Jun Tao. 2017. An unsupervised approach to inferring the localness of people using incomplete geotemporal online check-in data. ACM Trans. Intell. Syst. Technol. 8, 6, Article 80 (August 2017), 18 pages.

DOI: http://dx.doi.org/10.1145/3022471

\section{INTRODUCTION}

Two recent technical trends have changed people's daily lives fundamentally: the advent of online social media (Twitter, Facebook, Foursquare) and the proliferation of location-based sensors (e.g., GPS sensors). The combination of them has resulted in location-based social network (LBSN) services. In those services, people check in at

This material is based on work supported by the National Science Foundation under grants CBET-1637251, CNS-1566465, and IIS-1447795, and the Army Research Office under grant W911NF-16-1-0388. The views and conclusions contained in this document are those of the authors and should not be interpreted as representing the official policies, either expressed or implied, of the Army Research Office or the U.S. Government. The U.S. Government is authorized to reproduce and distribute reprints for Government purposes notwithstanding any copyright notation here on.

Authors' addresses: C. Huang, 100 University Village Apt A, Notre Dame, IN, 46556; email: chuang7@nd.edu; D. Wang, 214 B Cushing, University of Notre Dame, Notre Dame, IN, 46556; email: dwang5@nd.edu; J. Tao, 2007 Coachmans Trail, South Bend, IN, 46637; email: Jtao1@nd.edu.

Permission to make digital or hard copies of part or all of this work for personal or classroom use is granted without fee provided that copies are not made or distributed for profit or commercial advantage and that copies show this notice on the first page or initial screen of a display along with the full citation. Copyrights for components of this work owned by others than ACM must be honored. Abstracting with credit is permitted. To copy otherwise, to republish, to post on servers, to redistribute to lists, or to use any component of this work in other works requires prior specific permission and/or a fee. Permissions may be requested from Publications Dept., ACM, Inc., 2 Penn Plaza, Suite 701, New York, NY 10121-0701 USA, fax +1 (212) 869-0481, or permissions@acm.org.

(c) 2017 ACM 2157-6904/2017/08-ART80 $\$ 15.00$

DOI: http://dx.doi.org/10.1145/3022471 
the venues they visited and share their check-in data through online social network services. Examples of such services include Foursquare, BrightKite, Citysense, GyPSii, and MobiLuck. Millions of users have already adopted LBSN services, and their checkin points become an important open data source for a city to develop crowdsourcingbased smart city applications (intelligent transportation, urban infrastructure monitoring, geotagging, crowdsensing, etc.) [Wang and Huang 2015; Cardone et al. 2013; Wang et al. 2014b].

Along with this trend, an important problem is to infer the localness of people, where the goal is to classify people who are local residents in a city from people who visit the city by analyzing online check-in points that are contributed by online users. Solving this problem will allow for (i) inferring different activity/mobility patterns of local and nonlocal people in a city, and (ii) identifying the venues that are more likely to attract local or nonlocal people, respectively. Such information is critical for many locationbased applications, such as ads targeted for local business [Provost et al. 2009; Ahmed et al. 2011], urban planning [Ratti et al. 2006; Gonzalez et al. 2008], and localized news recommendations [White et al. 2009; Bennett et al. 2012].

A city may have a record of its local residents through census. However, online checkin data are distinct from census data in several ways. First, the census data is typically not publicly available or just includes some aggregated statistics and thus cannot be used for the location-based applications mentioned earlier. Second, the census data does not include the venues that individuals visit in their daily lives and hence is not helpful in understanding the activity/mobility patterns or identifying hot spots visited by local/nonlocal people in a city. Third, many people do not put their real names and home locations in their profiles on LBSN services, making it difficult (if not impossible) to map their LBSN accounts to the census information.

Supervised learning approaches have been developed to infer the location of people in a city by assuming the availability of high-quality training datasets with complete geotemporal information [Li et al. 2012b; Backstrom et al. 2010; Cheng et al. 2010, 2011; Li et al. 2012a]. However, such assumption does not always hold in practice for a couple of reasons. First, many people do not feel comfortable to upload their localness or home location information to the online services for privacy concerns [Ma et al. 2013]. Second, many LBSNs do not share all of their collected data with the general public [Zhang and Chow 2015]. Therefore, only a portion of the user's check-in points are available for analysis, and we refer to such data as geotemporal incomplete data. In particular, we define "incomplete geotemporal check-in point data" in this article to be the check-in traces that users share on LBSNs.

A few technical challenges exist to solve the problem of localness inference. First, the online check-in trace is incomplete and sparse: we cannot expect a person to check in at every place in the city (e.g., he or she either forgets to check in or intentionally chooses not to do so due to privacy concerns). Second, the collected data is "noisy": both local people and nonlocal visitors can check in at the same venue. There is no clear difference between the check-in points provided by different groups of people.

In this article, we develop an unsupervised learning approach to find local people in a city (Figure 1). In particular, we develop an incomplete geotemporal expectation maximization (IGT-EM) scheme, which incorporates a set of hidden variables to represent the localness of people and a set of estimation parameters to represent the likelihood of venues to attract local and nonlocal people, respectively. Our solution can identify the localness of people without prior knowledge on the people and the venues they visited. Additionally, we implement a parallel IGT-EM algorithm by leveraging the computing power of a graphic processing unit (GPU) that consists of 2,496 cores. In the evaluation, we compared our new approach with the existing solutions through four real-world case studies using data from New York City, Chicago, Boston, and Washington DC. The results showed that our approach can identify the local people and 


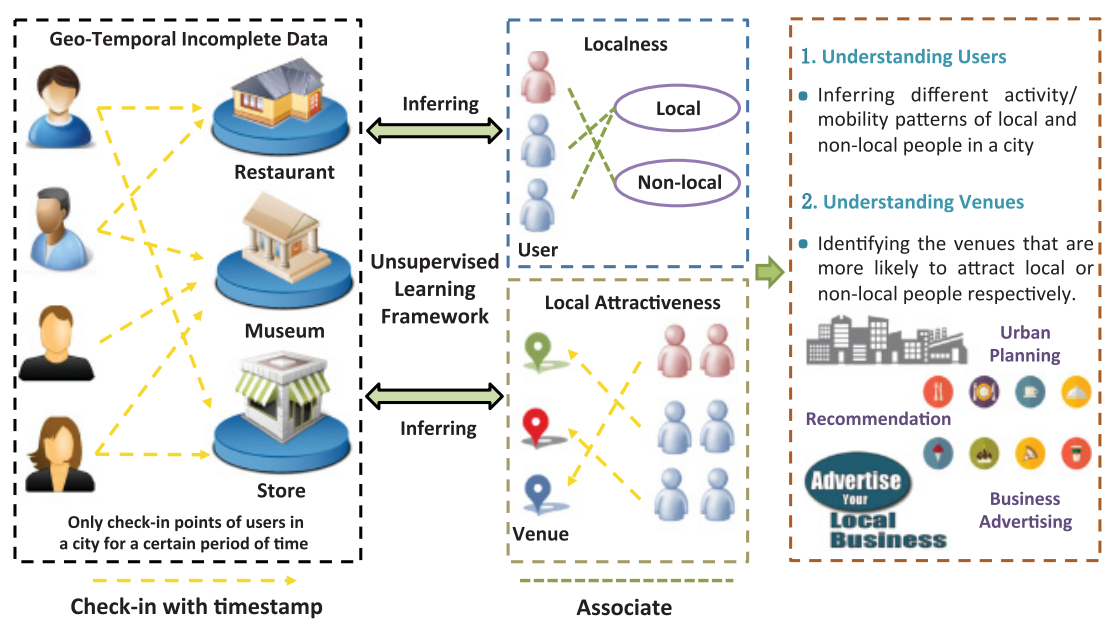

Fig. 1. Overview of the IGT-EM framework.

significantly outperform the compared baselines in estimation accuracy and execution time.

We summarize the main contributions of this article as follows:

-In this article, we address the problem of inferring the localness of people using an unsupervised approach based on incomplete geotemporal data.

-We develop a new approach to identify the local people without the prior knowledge on the people and the venues they visited (Section 4).

-We implement a parallel IGT-EM scheme that can run on GPU processors and demonstrate that the parallel algorithm runs a few orders of magnitude faster than its sequential counterpart (Section 5).

-We evaluate the estimation accuracy and execution time of the IGT-EM scheme through four real-world case studies using data from Foursquare. The IGT-EM scheme is shown to significantly outperform the compared baselines in identifying the localness of people (Section 6).

A preliminary version of this work was published in Huang and Wang [2016a]. This article significantly expands our previous work and makes new contributions from the following aspects. First, we developed a scalable framework of IGT-EM to implement our proposed scheme on a parallel platform (i.e., GPU), which can efficiently handle big data and is more suitable for large-scale online social network applications (Section 5). Second, we performed a set of experiments on a new dataset collected from the largest city in the United States (New York) and further evaluated the robustness and efficiency of our scheme in more real-world scenarios (Section 6). Third, we studied the performance of the IGT-EM scheme with respect to a set of key parameters in our model: the threshold for time length of check-in trace, the number of check-in points, and the density of the dataset. The new results verified the robustness of our model over different dimensions of the problem (Section 6). Finally, we also investigated the convergence of the IGT-EM scheme and presented the results of the convergence analysis of IGT-EM over different datasets (Section 6).

\section{RELATED WORK}

With the proliferation of the online social media and networks, some seminal work explores social network for user profiling [Lampe et al. 2007; Pfeil et al. 2009; Golbeck 
2009]. For example, Lampe et al. explored the relationship between profile structure and the number of friends to decide which profile elements are most likely to predict the friendship links on Facebook [Lampe et al. 2007]. Pfei et al. investigated the age differences and similarities in the use of the MySapce to explore potential differences in social capital among older people and teenagers [Pfeil et al. 2009]. Golbeck investigated features of profile similarity and their relationship to the user's trust by isolating profile features beyond the overall similarity and examined their solution on FilmTrust [Golbeck 2009]. However, none of the preceding works studied the problem of inferring the localness of people in a city. In contrast, this article focuses on the problem of identifying local people by using the incomplete geotemporal online social media data from a city.

Prior works exist on the topic of mining the location of people in a city from their online check-in data [Li et al. 2012b; Backstrom et al. 2010; Cheng et al. 2010, 2011; Li et al. 2012a; Jurgens 2013]. For example, Cheng et al. developed a statistical model to estimate the location of Twitter users by analyzing the key phrases used in tweets [Cheng et al. 2010, 2011]. They used a set of location-related phrases to train their model and associate those phrases with locations. Backstrom et al. estimated the location of a user by exploring the relationship between geography and friendship on online social networks [Backstrom et al. 2010]. Jurgens et al. developed a framework to infer locations of users by studying their spatial correlations and using a small number of ground truth locations as the initiation of its algorithm [Jurgens 2013]. Li et al. developed a novel analytical model to predict user's home location from leveraging the information from both social network and user-generated data [Li et al. 2012b]. They further extended their model to handle cases where users have multiple home locations [Li et al. 2012a]. However, the preceding solutions all need sufficient training data to build up their models and generate accurate estimation results and hence are all supervised learning approaches. On the contrary, this article takes an unsupervised learning approach to infer the localness of people without prior knowledge on the people and the venues they visited.

A category of location-based recommendation systems also bear a resemblance to our work [Yin et al. 2013; Chen et al. 2015; Park et al. 2007; Ramaswamy et al. 2009; Kodama et al. 2009]. For example, Yin et al. built a recommendation system that offers a particular user a set of venues or events by considering the user's personal interest and local preference [Yin et al. 2013]. Furthermore, Chen et al. developed a pointof-interest (POI) recommendation system to find the top-K location category-based POI recommendation by considering the information coverage in the recommendation process [Chen et al. 2015]. Park et al. developed a recommendation system for venues by exploring the visitor's profile information (e.g., location, age, or cuisine preferences) [Park et al. 2007]. Ramaswamy et al. proposed a location recommendation system that leverages a user's social affinity [Ramaswamy et al. 2009]. Kodama et al. designed an approach to recommend items to a user by taking into account his current location and preferences [Kodama et al. 2009]. In contrast, we focus on solving the problem of inferring the localness of people using LBSN data, which could be used in many recommendation systems, such as the ones discussed earlier.

\section{MODEL}

In this section, we describe the model we used to infer the localness of people in a city using incomplete geotemporal data. Let us consider an LBSN application where a set of users $U_{1}, U_{2}, \ldots, U_{Y}$ generate check-in points in a set of venues $V_{1}, V_{2}, \ldots, V_{X}$ in a city. Let $V_{i}$ represent the $i^{t h}$ venue and $U_{j}$ represent the $j^{\text {th }}$ user. We introduce a hidden variable $Z$ for each user to indicate the localness of that user. For example, $Z_{j}=1$ when 
the user $U_{j}$ is local and $Z_{j}=0$ if he or she is not. We also define a check-in matrix $V U$ : $V U_{i, j}=1$ indicates that the user $U_{j}$ checks in at venue $V_{i}$ and $V U_{i, j}=0$ otherwise.

We define the time length of a user's check-in trace as the time difference between the earliest and latest check-in points contributed by the user in the dataset. It varies from one person to another. We further define a time vector $T$, and the element $t_{j}$ is the time length of the check-in trace from user $U_{j}$. In this article, we explicitly consider both venue and time information and formulate an optimization problem to estimate the localness of people.

We then define $a_{i}$ as the local attractiveness of a venue $V_{i}$. Formally $a_{i}$ is given by

$$
a_{i}=P\left(U_{j}=1 \mid V U_{i, j}=1\right) .
$$

We define $a_{i}^{k}$ as the local attractiveness of $V_{i}$ to attract local people whose time length of check-in trace lasts for $k$ days. Formally, $a_{i}^{k}$ is given by

$$
a_{i}^{k}=P\left(U_{j}=1 \mid V U_{i, j}=1, t_{j}=k\right) .
$$

Therefore,

$$
a_{i}=\sum_{k=1}^{K} a_{i}^{k} \times \frac{r_{i}^{k}}{r_{i}} k=1, \ldots, K,
$$

where $r_{i}^{k}=P\left(V U_{i, j}=1, t_{j}=k\right)$. For each venue, $r_{i}^{k}$ can be computed by counting and dividing the frequencies of users check-in points at $V_{i}$ using the check-in matrix $V U$ and the time vector $T$. Note that $r_{i}=\Sigma_{k=1}^{K} r_{i}^{k}$.

We further define $b_{i, k}$ and $c_{i}^{k}$ as follows:

$$
\begin{aligned}
b_{i}^{k} & =P\left(V U_{i, j}=1, t_{j}=k \mid U_{j}=1\right), \\
c_{i}^{k} & =P\left(V U_{i, j}=1, t_{j}=k \mid U_{j}=0\right) .
\end{aligned}
$$

Following the Bayes theorem, we can obtain the relation between $b_{i}^{k}, c_{i}^{k}$ and $a_{i}^{k}, r_{i}^{k}$ as follows:

$$
b_{i}^{k}=\frac{a_{i}^{k} \times r_{i}^{k}}{l} c_{i}^{k}=\frac{\left(1-a_{i}^{k}\right) \times r_{i}^{k}}{1-l},
$$

where $l$ represents the probability that a randomly chosen user is local.

Therefore, we can formulate the problem of identifying local people using incomplete geotemporal data studied as a constraint maximum likelihood estimation (MLE) problem: given only the check-in matrix $V U$ and time vector $T$, our goal is to compute the following:

$$
\begin{aligned}
& \forall j, 1 \leq j \leq Y: P\left(U_{j}=1 \mid V U, T\right) \\
& \forall i, 1 \leq i \leq X: P\left(U_{j}=1 \mid V U_{i, j}=1\right)
\end{aligned}
$$

\section{SOLUTION}

In this section, we solve the problem of local people identification formulated in Section 3 by developing an IGT-EM algorithm.

\subsection{Background}

Expectation maximization (EM) is a commonly used optimization technique for the MLE problem where the model contains hidden variables that cannot be directly observed from the data. Specifically, it contains two steps:

$$
\text { E-step: } Q\left(\theta \mid \theta^{(n)}\right)=E_{Z \mid x, \theta^{(n)}}[\log L(\theta ; x, Z)],
$$




$$
\text { M-step: } \theta^{(n+1)}=\arg \max _{\theta} Q\left(\theta \mid \theta^{(n)}\right) \text {. }
$$

We solve the localness inference problem by developing an IGT-EM scheme. Specifically, the observed data $X$ in our problem is the check-in matrix $V U$ and the time period vector $T$. The estimation parameter vector is defined as $\theta=$ $\left(b_{1}^{k}, b_{2}^{k}, \ldots, b_{X}^{k} ; c_{1}^{k}, c_{2}^{k}, \ldots, c_{X}^{k} ; l\right)$, where $k=1,2, \ldots, K ; b_{i}^{k}$ and $c_{i}^{k}$ are defined in Equation (4). We further introduce a vector of hidden variables $Z$ to indicate whether a user is local or not, particularly the variable $z_{j}$ for user $U_{j}$ such that $z_{j}=1$ if $U_{j}$ is local and $z_{j}=0$ otherwise. Finally, we define a set of indication variables $t_{j}^{k}$ such that $t_{j}^{k}=1$ if $t_{j}=k$ in time period vector $T$ and $t_{j}^{k}=0$ otherwise.

The likelihood function for IGT-EM is as follows:

$$
\begin{aligned}
& L(\theta ; X, Z)=\operatorname{Pr}(X, Z \mid \theta) \\
& =\prod_{j=1}^{Y}\left\{\prod_{i=1}^{X} \prod_{k=1}^{K}\left(\beta_{i, j}^{k}\right)^{V_{i} U_{j} \times t_{j}^{k}} \times\left(\beta_{i, j}^{k}\right)^{\left(1-V_{i} U_{j}\right)} \times l \times z_{j}\right. \\
& \left.\quad+\prod_{i=1}^{X} \prod_{k=1}^{K}\left(\beta_{i, j}^{k}\right)^{V U_{i, j} \times t_{j}^{k}} \times\left(\beta_{i, j}^{k}\right)^{\left(1-V U_{i, j}\right)} \times(1-l) \times\left(1-z_{j}\right)\right\},
\end{aligned}
$$

where $V U_{i, j}=1$ when user $U_{j}$ visits venue $V_{i}$ and 0 otherwise. The $\beta_{i}^{k}$ are defined as follows:

$$
\beta_{i, j}^{k}= \begin{cases}b_{i}^{k} & \text { if } V U_{i, j}=1, t_{j}^{k}=1, z_{j}=1 \\ \left(1-\sum_{k=1}^{K} b_{i}^{k}\right) & \text { if } V U_{i, j}=0, t_{j}^{k}=1, z_{j}=1 \\ c_{i}^{k} & \text { if } V U_{i, j}=1, t_{j}^{k}=1, z_{j}=0 \\ \left(1-\sum_{k=1}^{K} c_{i}^{k}\right) & \text { if } V U_{i, j}=0, t_{j}^{k}=1, z_{j}=0 .\end{cases}
$$

\subsection{Incomplete Geotemporal Expectation Maximization}

We can derive the E-step as follows:

$$
\begin{aligned}
& Q\left(\theta \mid \theta^{(n)}\right)=E_{Z \mid X, \theta^{(n)}}[\log L(\theta ; X, Z)] \\
& =\sum_{j=1}^{Y}\left\{\operatorname{Pr}\left(z_{j}=1 \mid X_{j}, \theta^{(n)}\right) \times\left[\sum_{i=1}^{X} \sum_{k=1}^{K}\left(V U_{i, j} \times t_{j}^{k}\right) \times \log \beta_{i, j}^{k}+\left(1-V U_{i, j}\right) \times \log \beta_{i, j}^{k}+\log l\right]\right. \\
& \left.+\operatorname{Pr}\left(z_{j}=0 \mid X_{j}, \theta^{(n)}\right) \times\left[\sum_{i=1}^{X} \sum_{k=1}^{K}\left(V U_{i, j} \times t_{j}^{k}\right) \times \log \beta_{i, j}^{k}+\left(1-V U_{i, j}\right) \times \log \beta_{i, j}^{k}+\log (1-l)\right]\right\} .
\end{aligned}
$$

Note that in the $Q$ function, the estimation parameters are represented by $\beta_{i, j}^{k}$, which is defined in Equation (10); $\beta_{i, j}^{k}$ represents different parameters under different conditions. 


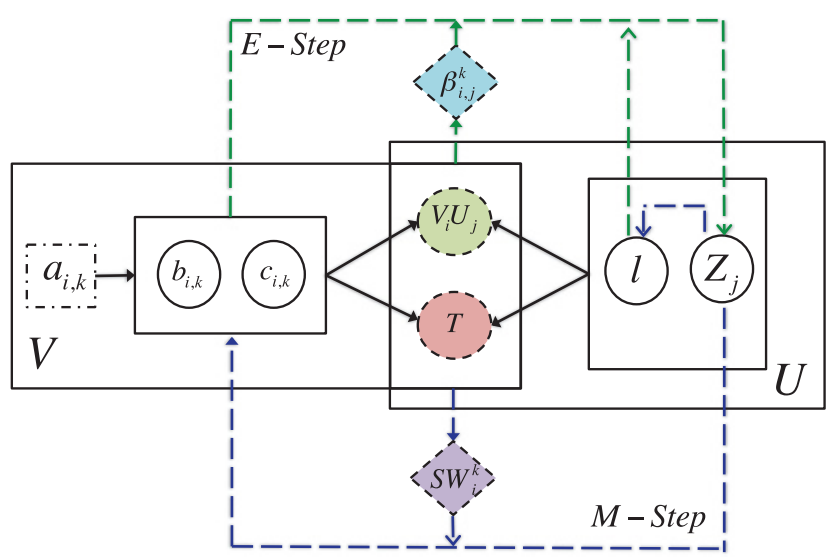

Fig. 2. IGT-EM scheme.

To drive the M-step, we set partial derivatives of $Q\left(\theta \mid \theta^{(n)}\right)$ given by Equation (11) with respect to $\theta$ to 0 to get the optimal $\theta^{*}$. Specifically, we get the solutions of $\frac{\partial Q}{\partial b_{i}^{k}}=0$, $\frac{\partial Q}{\partial c_{i}^{k}}=0$, and $\frac{\partial Q}{\partial l}=0$ in each iteration. The optimal estimation of the parameters for the next iteration (i.e., $\left(b_{i}^{k}\right)^{(n+1)},\left(c_{i}^{k}\right)^{(n+1)}$, and $\left.(l)^{(n+1)}\right)$ are as follows:

$$
\begin{aligned}
& \left(b_{i}^{k}\right)^{(n+1)}=\frac{\Sigma_{j \in S W_{i}^{k}} \operatorname{Pr}\left(z_{j}=1 \mid X_{j}, \theta^{(n)}\right)}{\Sigma_{j \in U} \operatorname{Pr}\left(z_{j}=1 \mid X_{j}, \theta^{(n)}\right)} \\
& \left(c_{i}^{k}\right)^{(n+1)}=\frac{\Sigma_{j \in S W_{i}^{k}}\left(1-\operatorname{Pr}\left(z_{j}=1 \mid X_{j}, \theta^{(n)}\right)\right)}{\Sigma_{j \in U}\left(1-\operatorname{Pr}\left(z_{j}=1 \mid X_{j}, \theta^{(n)}\right)\right)} \\
& (l)^{(n+1)}=\frac{\Sigma_{j \in U} \operatorname{Pr}\left(z_{j}=1 \mid X_{j}, \theta^{(n)}\right)}{|U|},
\end{aligned}
$$

where $S W_{i}^{k}$ is the group of users who check in at venue $U_{i}$ and the time length of their check-in points is $k$ days in a city. $U$ is the set of all users. The E- and M-steps of the IGT-EM scheme are shown in Figure 2.

\section{PARALLEL IGT-EM}

To further improve computing performance, we design a parallel implementation of the IGT-EM scheme on a GPU that uses the Compute Unified Device Architecture (CUDA) programming model [NVIDIA 2008]. The GPU has emerged as a new computing platform for many compute-intensive applications. CUDA is a parallel programming model invented by NVIDIA. In CUDA, a kernel is defined as a grid of thread blocks, and a thread of execution is the smallest unit in the parallelization. In the parallelization process, each node (called a thread node) will take care of a part of the whole computation task, and users need to specify a set of kernels to parallelize the computation task.

In this work, we implement a parallel version of the IGT-EM scheme on a GPU platform to improve its computation efficiency for large-scale datasets. Although there exist prior studies on parallel implementation of the EM algorithm, several challenges exist to implement parallel IGT-EM: (i) the memory of graphics cards is limited, so we need to design efficient strategies to handle the large-scale datasets from the LBSN on the GPU, and (ii) we need to design a mechanism to distribute the computation task of various estimation parameters and hidden variables of IGT-EM to different threads 
in an efficient way. To address these challenges, we designed the parallel IGT-EM based on the MLE model developed in this article and optimized our implementation using the following techniques: (i) we set the variables used in each thread as local variables instead of global variables given the fact that it costs more time to access global memory than local memory, and (ii) we replaced the original conditional branch in the implementation of IGT-EM algorithm with direct index in the corresponding arrays, which helps optimize the control flow and reduce the waiting time of threads. The preceding optimization leads to significant execution time improvement achieved by IGT-EM, as shown in the next section. We summarize the parallel IGT-EM scheme in Algorithm 1.

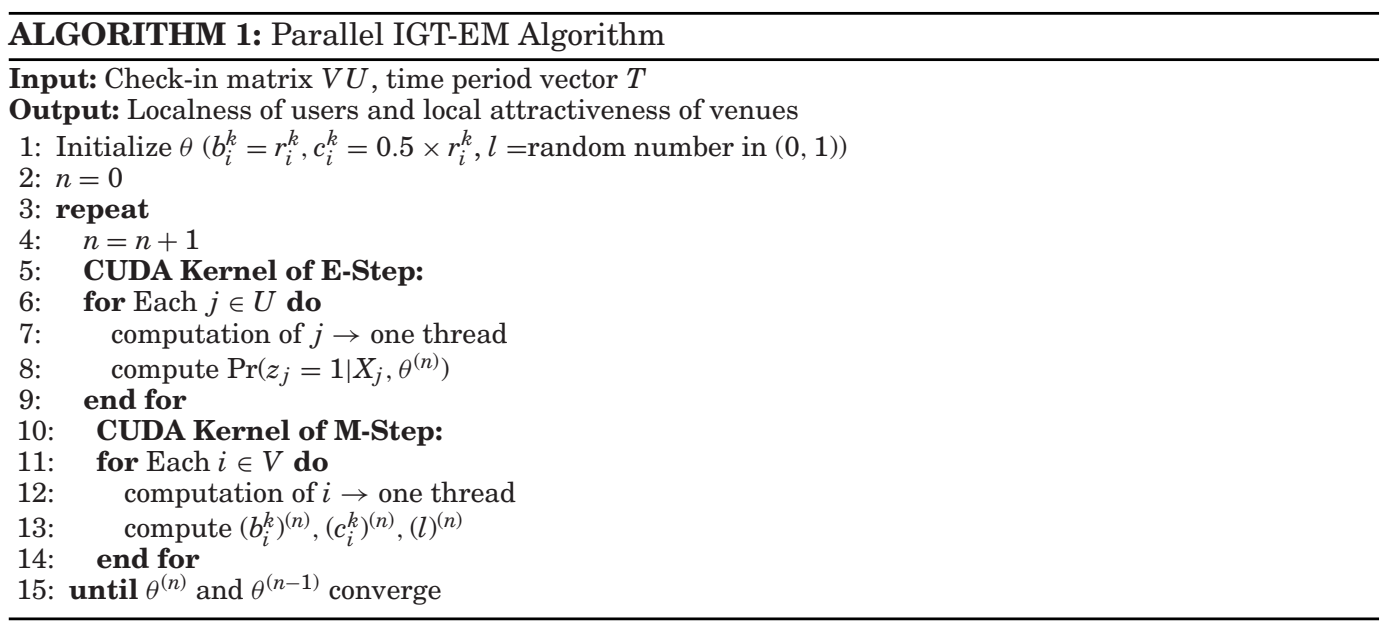

\section{EVALUATION}

In this section, we conduct experiments to study the performance of IGT-EM compared to a set of state-of-the-art baselines through four real-world case studies using data collected from Foursquare. We show that the IGT-EM scheme can classify local people from nonlocal people more accurately and efficiently than the compared baselines.

\subsection{Experimental Setups}

6.1.1. Dataset Statistics. In this work, we study the performance of our proposed scheme using data traces collected from Foursquare, a widely used LBSN. In the evaluation, we selected four data traces from cities in the United States with user home location information available: ${ }^{1}$ New York, Chicago, Washington, DC, and Boston. Figure 3 shows the venue maps of the four cities. ${ }^{2}$ Figures 4 and 5 further show the distributions of check-in points per venue (density) and per users (frequency) in the four datasets, respectively. In these figures, we can observe that the check-in points of the datasets are "incomplete" in the sense that check-in points at many venues are sparse and that many users check in at venues with a low frequency. The statistics of the four data traces are summarized in Table I.

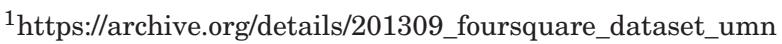

${ }^{2}$ Many venues are so close together that they overlap with each other in the map.
} 


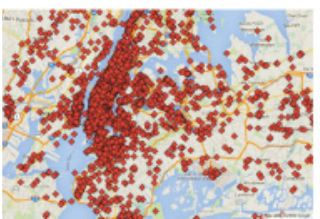

(a) New York

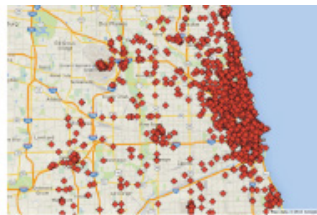

(b) Chicago

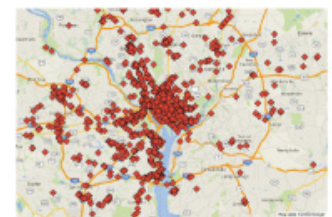

(c) Washington D.C

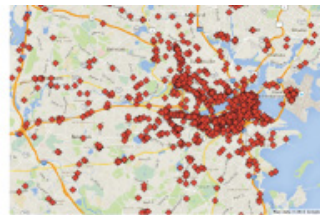

(d) Boston

Fig. 3. Maps of venues in four cities.

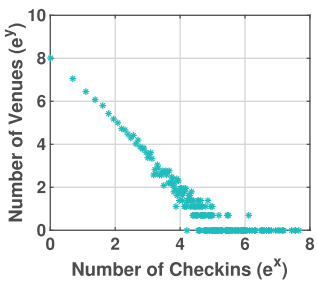

(a) New York

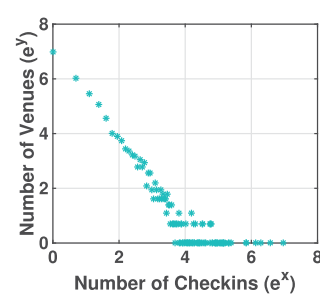

(b) Chicago

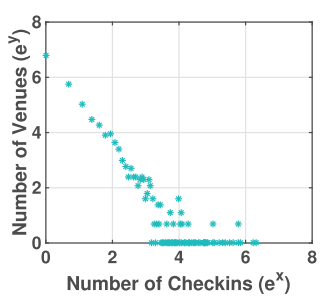

(c) Washington D.C

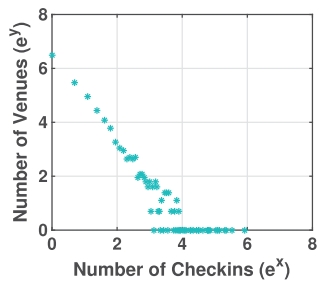

(d) Boston

Fig. 4. Distribution of check-in points per venue (density).

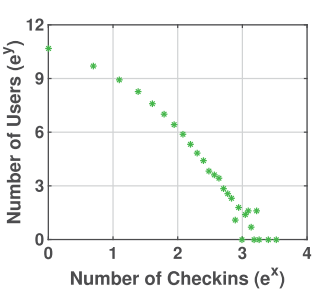

(a) New York

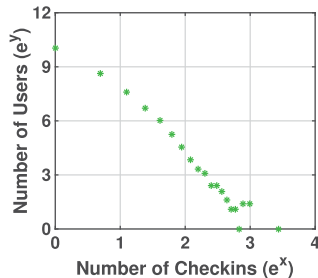

(b) Chicago

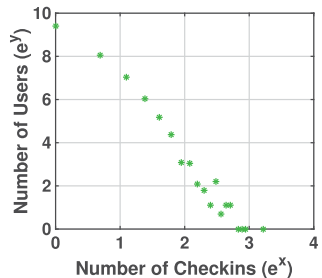

(c) Washington D.C

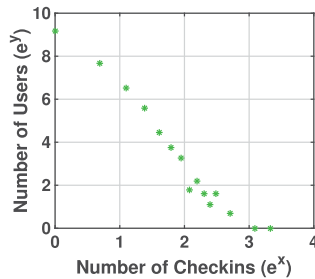

(d) Boston

Fig. 5. Distribution of check-in points per user (frequency).

Table I. Data Traces Statistics

\begin{tabular}{|l|c|c|c|l|}
\hline Data Trace & New York & Chicago & Washington, DC & Boston \\
\hline Number of Users & 75,680 & 31,965 & 17,231 & 12,946 \\
\hline Number of Venues & 7,663 & 2,529 & 1,932 & 1,478 \\
\hline Number of Check-Ins & 144,179 & 48,605 & 25,722 & 18,296 \\
\hline
\end{tabular}

6.1.2. Data Preprocessing. We designed a few data preprocessing steps to generate the inputs to the IGT-EM scheme:

-Generating check-in matrix: We generate the $V U$ matrix by examining which user checks in at which venue. For example, if user $U_{j}$ makes a check-in point at venue $V_{i}$, we set the element $V_{i} U_{j}$ in $V U$ to 1 and 0 otherwise.

- Generating time vector: For simplicity, we divide all users into two sets based on the time length of their check-in trace. For example, if the time length of a user's checkin points (i.e., the time difference between the earliest and latest check-in points) is larger than a predefined threshold (we used 10 days in our experiment), we set the corresponding element $t_{j}$ in vector $T$ as 1 . Otherwise, we set the $t_{j}$ as 0 .

The preceding preprocessing steps generated all of the inputs (i.e., $V U$ matrix and $T$ vector) that are needed for the IGT-EM scheme. 


\subsection{Evaluation of Our Scheme}

6.2.1. Baselines. We compared the IGT-EM scheme to the following state-of-the-art techniques:

-Regular-EM: This uses a basic EM approach (that does not consider the time length of check-in trace) to solve the problem of inferring the localness of people [Wang et al. 2012]. Particularly, the input to the regular-EM algorithm is only venue-user matrix $V U$ and does not consider time vector $T$ in the estimation model.

$-M L P$ : This is a supervised learning scheme that leverages the location information of a user's online friends to infer the locations of the target user [Li et al. 2012a]. In particular, it estimates a user's location by taking average of home coordinates of people who have an online social connection with the user.

-FM: This is another supervised learning approach that solves the problem by leveraging the home locations of the people who have a similar venue visit pattern as the target users [Backstrom et al. 2010].

-FL: This argues the MLP approach by explicitly considering the social tie strength between users [McGee et al. 2013].

- HLI: This develops a machine learning approach that estimates a user's location by assuming that users who have check-in points during the evening in a city are more likely to be local users [Hu et al. 2015].

$-L P$ : This presents an algorithm that leverages the geographic distribution of an individual's ego network (i.e., social network in one-hop) to infer his or her locations [Jurgens 2013]. In particular, it estimates the location of a user by taking the average of home coordinates of his or her online social friends in the ego network.

-Voting: This decides the localness of a user by assuming that local people visit more venues than nonlocal people.

6.2.2. Results. In the evaluation, we use the home location of users as the ground truth information to decide whether a user is local or not. For example, if the user's home location is X miles away from the center of the city, the user is considered to be local. We also changed the value of $\mathrm{X}$ in our experiments to study the performance of our scheme and other baselines. One should note that we only used the ground truth information for the evaluation purpose and did not use it as the input to our IGT-EM scheme.

Figure 6 shows the results of IGT-EM and other baselines in the New York City dataset. IGT-EM is observed to clearly outperform the compared baselines: it classifies local people from nonlocal people with smallest false positives and false negatives. It also has a more accurate estimation on how attractive a venue is for local people compared to other schemes. For example, IGT-EM outperforms the best baseline by $12 \%$ and $8 \%$ on accuracy and F1-measure, respectively. We also observe that the performance gain of IGT-EM is consistent when we change the values of X. Figure 7 shows the results of the Chicago data trace. Similar results are observed: IGT-EM continues to outperform all baselines in both inferring the localness of people and the estimation error on the local attractiveness of venues. The results of the Washington, DC, and Boston data traces are shown in Figures 8 and 9, respectively. In these two figures, similar evaluation results can be observed: IGT-EM is the best-performed scheme over all evaluation metrics. The preceding results show that IGT-EM can effectively identify the localness of people in a city and achieves significant performance gains compared to state-of-the-art techniques.

To investigate the effect of check-in time length (the time difference between the earliest and latest check-in points of a user) threshold that we used to generate the time vector $T$, we studied the performance of our proposed IGT-EM scheme by varying 


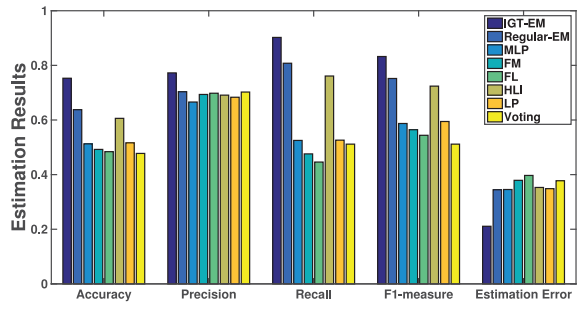

(a) Distance Threshold $=15$ miles

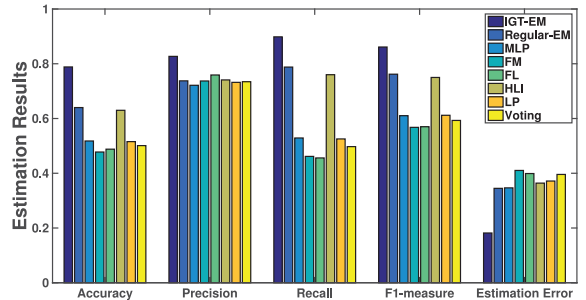

(c) Distance Threshold $=50$ miles

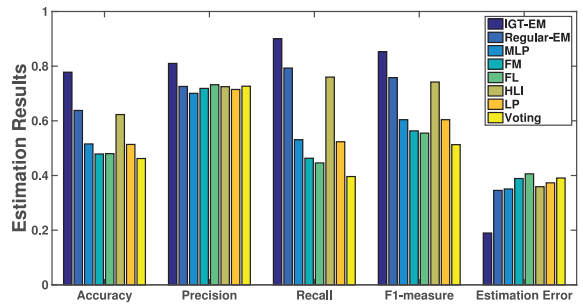

(b) Distance Threshold $=30$ miles

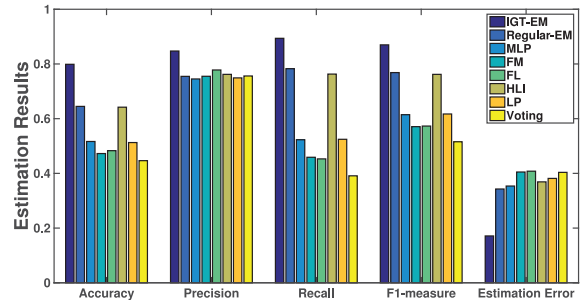

(d) Distance Threshold $=100$ miles

Fig. 6. Estimation results on the New York data trace.

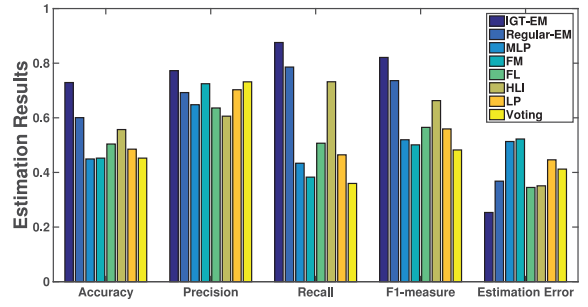

(a) Distance Threshold $=15$ miles

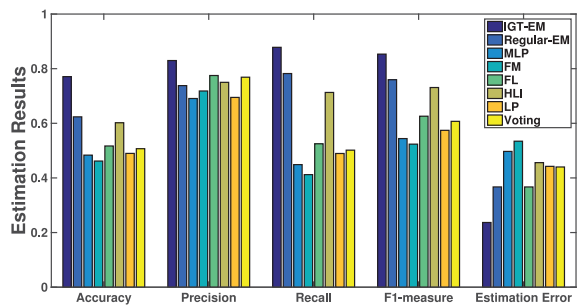

(c) Distance Threshold $=50$ miles

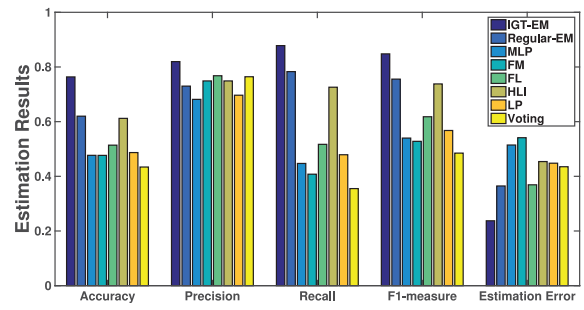

(b) Distance Threshold $=30$ miles

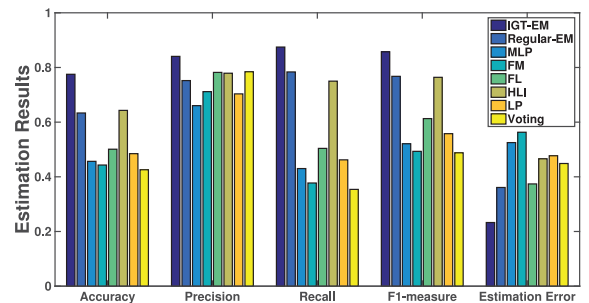

(d) Distance Threshold $=100$ miles

Fig. 7. Estimation results on the Chicago data trace.

the threshold of check-in time length (i.e., 6 days, 8 days, 10 days, 12 days, 14 days, and 16 days). In particular, IGT-EM- $t$ represents the corresponding IGT-EM scheme with $t$ days as the check-in time length threshold value. The evaluation results on all data traces are presented in Figure 10. We observe that the performance of the IGT-EM scheme is robust to the values of $t$ in the evaluated range. The reason is that the ratio of nonlocal to local users in the evaluated threshold range (i.e., 6 to 16 days) is relatively 


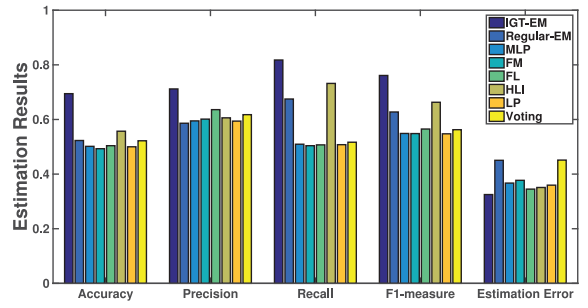

(a) Distance Threshold $=15$ miles

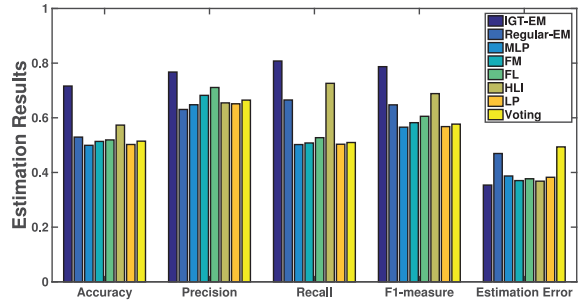

(c) Distance Threshold $=50$ miles

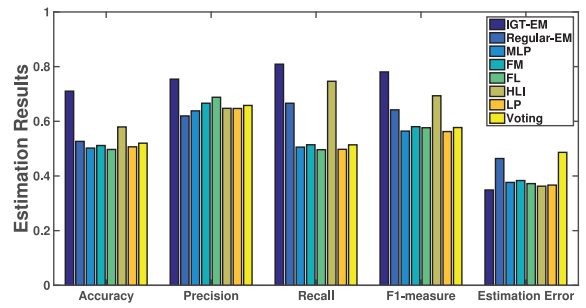

(b) Distance Threshold $=30$ miles

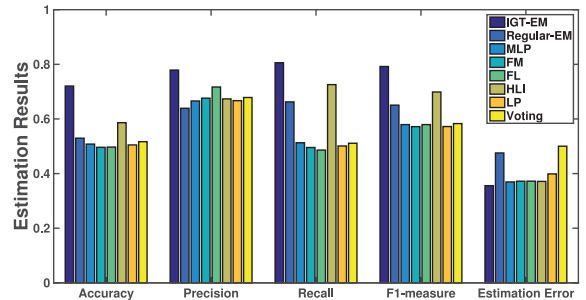

(d) Distance Threshold $=100$ miles

Fig. 8. Estimation results on the Washington, DC, data trace.

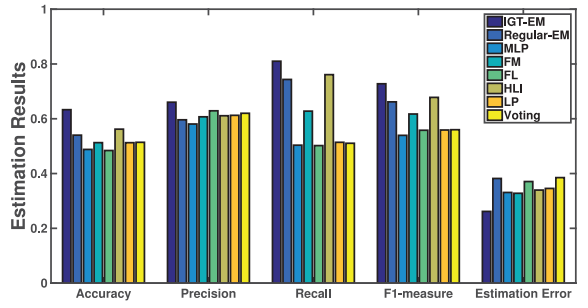

(a) Distance Threshold $=15$ miles

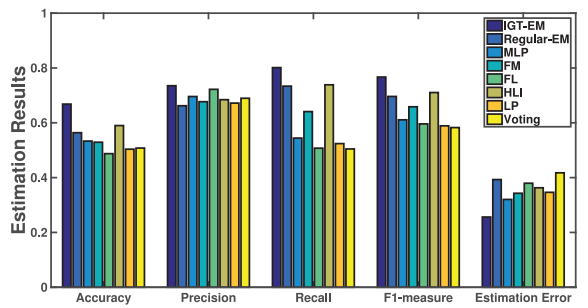

(c) Distance Threshold $=50$ miles

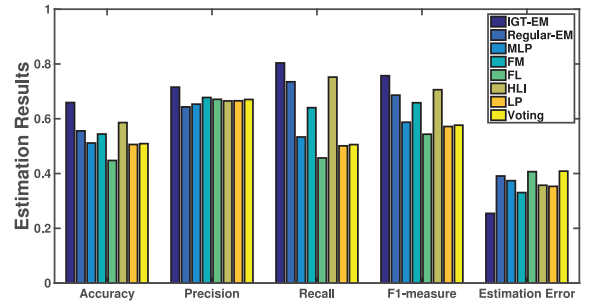

(b) Distance Threshold $=30$ miles

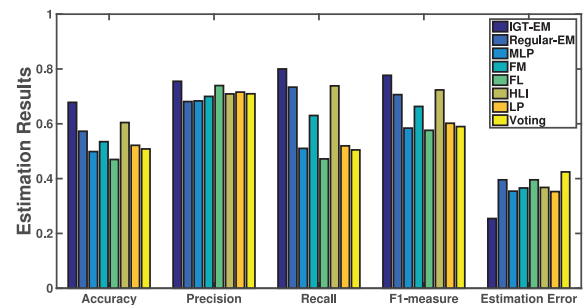

(d) Distance Threshold $=100$ miles

Fig. 9. Estimation results on the Boston data trace.

stable (i.e., 2 to 1.8), which leads to the insensitiveness of the IGT-EM scheme to the check-in length threshold.

We further investigate the scalability of the IGT-EM scheme in several different dimensions. We first compared the performance of IGT-EM scheme on four cities to 


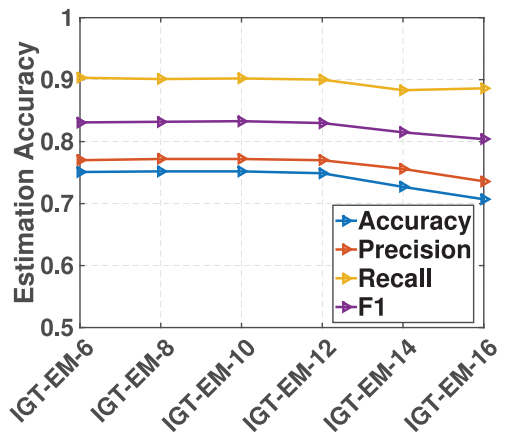

(a) New York Data Trace

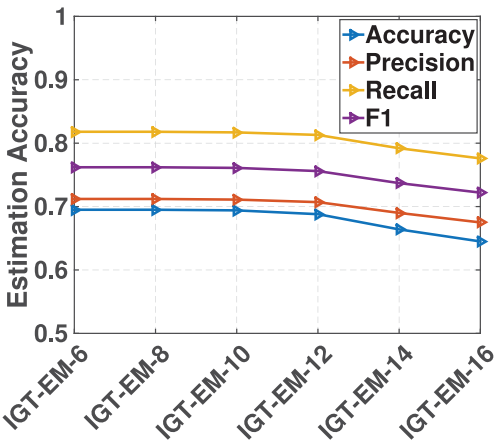

(c) Washington D.C Data Trace

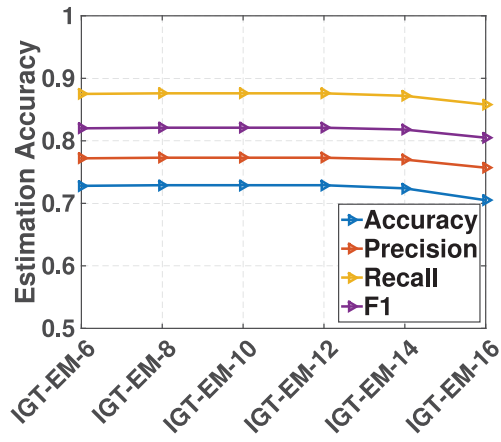

(b) Chicago Data Trace

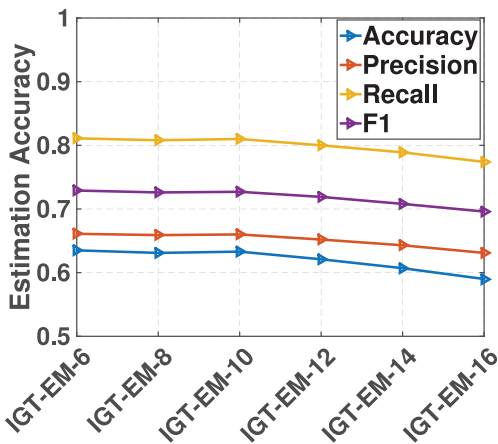

(d) Boston Data Trace

Fig. 10. Performance evaluation of IGT-EM- $t$.

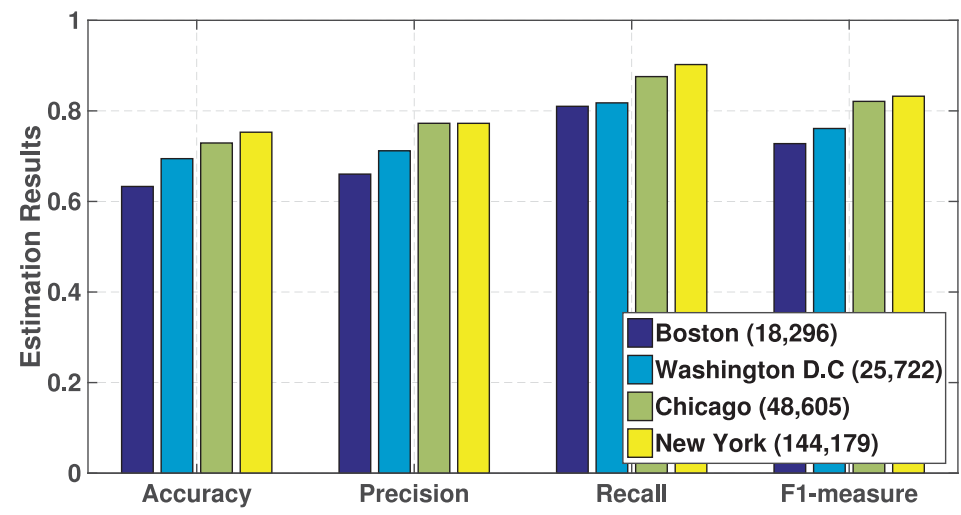

Fig. 11. Performance of IGT-EM on four cities with different numbers of check-ins.

different numbers of check-in points (i.e., New York City, Chicago, Washington, DC, and Boston). The results are reported in Figure 11. We can observe that the performance of IGT-EM improves as the number of check-in points increases. The reason is intuitive: more check-in points provide more evidence for IGT-EM to accurately infer the localness of the users. Furthermore, we also investigate the effect of check-in point density (number of check-in points per venue) and frequency (number of check-in points per 


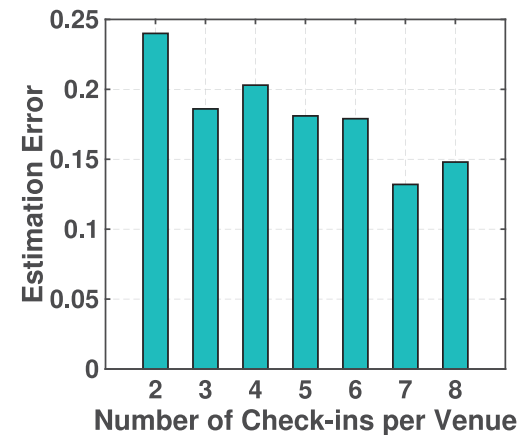

(a) New York Data Trace

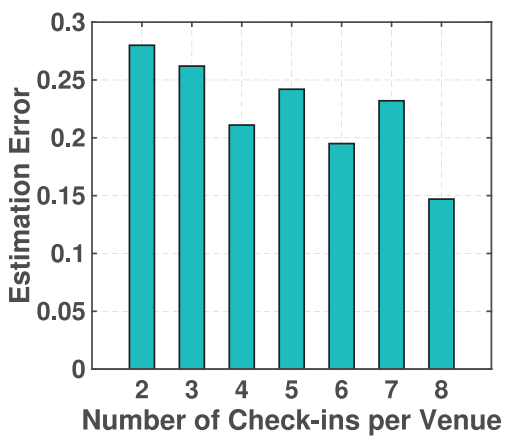

(b) Boston Data Trace

Fig. 12. Performance of IGT-EM by varying the check-in points per venue.

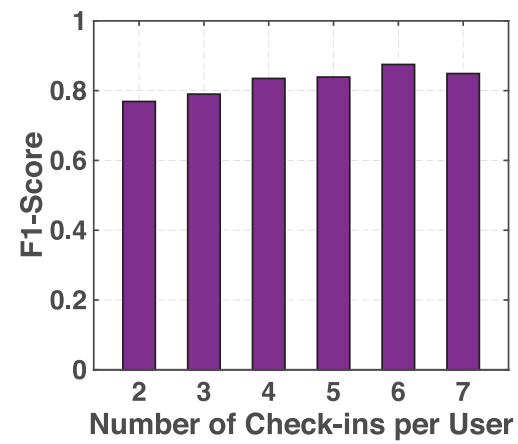

(a) New York Data Trace

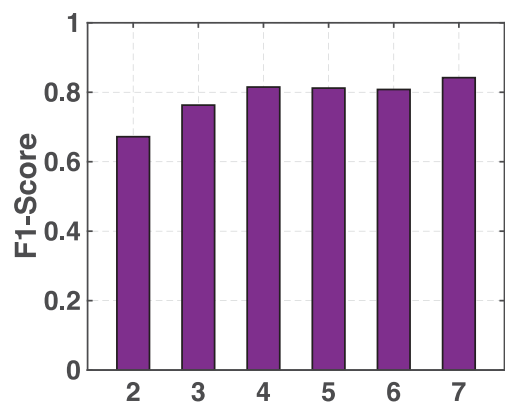

Number of Check-ins per User

(b) Boston Data Trace

Fig. 13. Performance of IGT-EM by varying the check-in points per user.

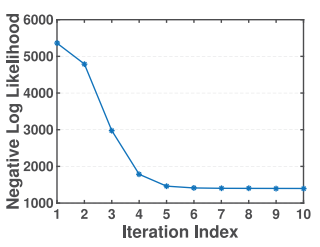

(a) New York Data Trace

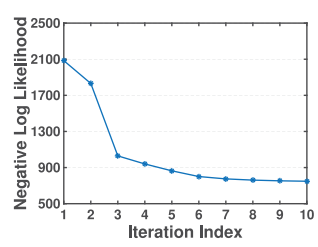

(b) Chicago Data Trace

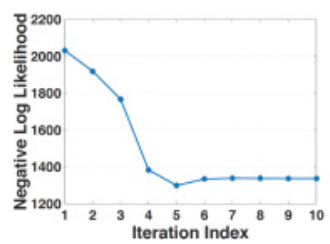

(c) Washington D.C Data Trace

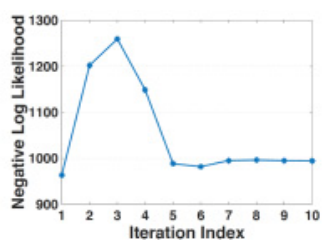

(d) Boston Data Trace

Fig. 14. Convergence analysis of IGT-EM.

user) on the performance of the IGT-EM scheme. The results are reported in Figures 12 and 13, respectively. In particular, we choose the city with the largest number of checkin points (New York City) and the city with the smallest number of check-in points (Boston) in our datasets as two typical examples in the reported results. We observe that the performance of IGT-EM in general improves as the number of check-in points per venue and per user increases, respectively.

We also investigated the convergence of the IGT-EM scheme on the four data traces; the results are presented in Figure 14. The figure shows the negative likelihood function with respect to the number of iterations of the IGT-EM scheme. We observe that the IGT-EM scheme converges quickly on all data traces. 
Table II. Execution Time Comparison

\begin{tabular}{|l|c|c|c|c|}
\hline Algorithms & New York $(\mathrm{s})$ & Chicago (s) & Boston (s) & Washington, DC (s) \\
\hline Parallel IGT-EM & $\mathbf{0 . 4 6}$ & $\mathbf{0 . 1 1 9}$ & $\mathbf{0 . 1 9 2}$ & $\mathbf{0 . 1 3 1}$ \\
\hline Sequential IGT-EM & 909.90 & 34.47 & 26.20 & 65.01 \\
\hline Regular-EM & 465.32 & 16.93 & 12.91 & 31.51 \\
\hline MLP & 163.25 & 19.37 & 17.53 & 2.88 \\
\hline FM & 266.09 & 4.15 & 17.53 & 13.71 \\
\hline FL & 277.45 & 30.04 & 46.03 & 24.18 \\
\hline HLI & 613.40 & 11.67 & 23.75 & 8.62 \\
\hline LP & 148.55 & 16.46 & 19.28 & 3.42 \\
\hline Voting & 21.69 & 0.62 & 0.47 & 1.25 \\
\hline
\end{tabular}

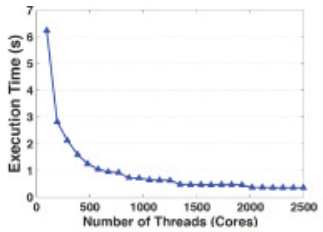

(a) New York Data Trace

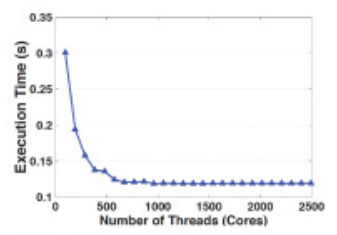

(b) Chicago Data Trace

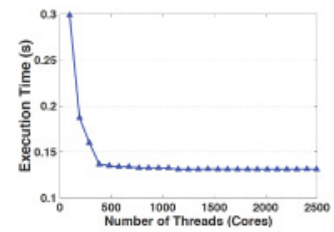

(c) Washington D.C Data Trace

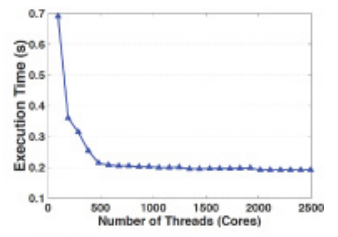

(d) Boston Data Trace

Fig. 15. Effects of number of threads on execution time.

Finally, we evaluate the efficiency of the parallel IGT-EM implementation discussed in Section 5. We implement the parallel IGT-EM on a computer with an NVIDIA GeForce GPU (2,496 cores and 1.25GHZ for each core, $4 \mathrm{~GB}$ memory). We compare the parallel IGT-EM with all baselines discussed earlier. We run the sequential IGI-EM (i.e., the IGT-EM scheme without parallel implementation) and other baselines on a regular lab computer ( 4 cores and $2 \mathrm{GHZ}$ for each core, $8 \mathrm{~GB}$ memory). Table II presents the execution time required by all algorithms on four data traces. We observe that the parallel IGT-EM runs several orders of magnitude faster than the sequential IGTEM and other baselines. The performance gain is more significant on the New York trace since it has more users, venues and check-in points. The efficiency of the parallel IGT-EM is achieved by leveraging the computation powers from thousands of cores in the GPU. Figure 15 shows the execution time of the parallel IGT-EM with respect to varying numbers of threads (cores). Observe that the execution time drops quickly as the number of cores increases (i.e., more computational tasks in the parallel IGT-EM run in parallel). We also examined the estimation performance of the parallel IGT-EM: the results are exactly the same as the ones shown in Figures 6 through 9. The reason is straightforward: the parallel IGT-EM is just a parallel implementation of the same IGT-EM algorithm we discussed in Section 4. We simply distribute the computation task of estimation parameters and hidden variables to different threads on different GPU cores, which should not affect the estimation performance (as verified by our experiments).

\section{DISCUSSION AND FUTURE WORK}

In this article, we developed an unsupervised learning framework to infer the localness of people in a city using incomplete geotemporal data. Some directions exist for future work.

We investigated the binary case on the localness of people: a person is either local or nonlocal. We also demonstrated that our scheme is insensitive to the distance threshold used to decide whether a person is local or not. However, it might also be interesting to 
know the exact distance between the user's home location and the center of the city. Our current MLE framework can be extended to solve such a problem [Wang et al. 2014a]. In our next step, we plan to extend the binary hidden variable that represents the localness of a person to a continuous variable that could represent the exact distance between the person's home location and the center of the city. The key challenge would be how to develop the likelihood function of the MLE problem that could generate a close-loop solution.

The four datasets used in the experiments were collected from large cities in the United States (New York, Chicago, Washington, DC, and Boston). It would be interesting to study the performance of our method in small cities. We expect that the performance of IGT-EM will be affected by the following factors in small cities: (i) there are fewer numbers of users and venues in small cities, so the IGT-EM algorithm is expected to converge faster, and (ii) small cities may have a different distribution of check-in points per venue and per user from large cities, which will affect the estimation accuracy of the IGT-EM scheme as well. Unfortunately, we do not have sufficient data from small cities in our current datasets to rigorously evaluate the performance of our model. Therefore, we leave this as an important direction to investigate further in a future study.

Our work also bears a resemblance to the works that estimate the locations of people by exploring the textual information from social media. For example, Cheng et al. developed a statistical model to estimate the location of Twitter users by analyzing the key phrases used in tweets [Cheng et al. 2010, 2013]. We can integrate the textual information into our IGT-EM scheme and further improve its estimation performance by exploring the content of a user's texts. For example, a user might use words or languages that are specific to a city (e.g., local dialect) in the comments that he or she submits together with the check-in points at the visited venues. In such context, our model could be extended to incorporate the textual features as additional latent variables to characterize the localness of users. We expect that this extension will further improve the estimation accuracy of our scheme.

The inputs to the IGT-EM scheme (i.e., $V U$ matrix and $T$ vector) can be readily obtained from location-based online social network services. This minimal requirement on prior/external knowledge makes the proposed method robust and generally applicable to different application scenarios [Huang et al. 2015; Huang and Wang 2016b; Marshall and Wang 2016; Marshall et al. 2016]. However, we might still be able to improve the performance of IGT-EM if additional information about users and venues is known to the application. For example, knowing some of a venue's local attractiveness a priori (e.g., the Statue of Liberty is more likely to attract tourists than local people), we can initialize the IGT-EM scheme with a better start point (compared to a random start point). This will greatly expedite the convergence process of the EM algorithm and improve the response time of IGT-EM in large cities. The key challenge is how to incorporate the additional information into the proposed model without sacrificing the rigidity of the analytical framework. The authors are actively working on the preceding extensions.

In summary, this work solves the problem of identifying the local people in a city by using incomplete online check-in points from location-based online social network services. We developed an IGT-EM scheme that classifies local people from nonlocal ones under a rigorous analytical framework. We also developed a parallel implementation of IGT-EM based on a GPU with 2,496 cores to improve computing performance. We studied the performance of our new approach through four real-world case studies using data from Foursquare. We demonstrated that our approach can accurately identify the local people in a city and can significantly outperform other state-of-the-art baselines. The results of our work are important because they can directly contribute 
to many crowdsourcing-based smart city applications where training data is difficult or expensive to obtain.

\section{REFERENCES}

Amr Ahmed, Yucheng Low, Mohamed Aly, Vanja Josifovski, and Alexander J. Smola. 2011. Scalable distributed inference of dynamic user interests for behavioral targeting. In Proceedings of the 17th ACM SIGKDD International Conference on Knowledge Discovery and Data Mining. ACM, New York, NY, 114-122.

Lars Backstrom, Eric Sun, and Cameron Marlow. 2010. Find me if you can: Improving geographical prediction with social and spatial proximity. In Proceedings of the 19th International Conference on World Wide Web. ACM, New York, NY, 61-70.

Paul N. Bennett, Ryen W. White, Wei Chu, Susan T. Dumais, Peter Bailey, Fedor Borisyuk, and Xiaoyuan Cui. 2012. Modeling the impact of short-and long-term behavior on search personalization. In Proceedings of the 35th International ACM SIGIR Conference on Research and Development in Information Retrieval. ACM, New York, NY, 185-194.

Giuseppe Cardone, Luca Foschini, Paolo Bellavista, Antonio Corradi, Cristian Borcea, Manoop Talasila, and Reza Curtmola. 2013. Fostering participation in smart cities: A geo-social crowdsensing platform. IEEE Communications Magazine 51, 6, 112-119.

Xuefeng Chen, Yifeng Zeng, Gao Cong, Shengchao Qin, Yanping Xiang, and Yuanshun Dai. 2015. On information coverage for location category based point-of-interest recommendation. In Proceedings of the 29th AAAI Conference on Artificial Intelligence.

Zhiyuan Cheng, James Caverlee, and Kyumin Lee. 2010. You are where you tweet: A content-based approach to geo-locating Twitter users. In Proceedings of the 19th ACM International Conference on Information and Knowledge Management. ACM, New York, NY, 759-768.

Zhiyuan Cheng, James Caverlee, and Kyumin Lee. 2013. A content-driven framework for geolocating microblog users. ACM Transactions on Intelligent Systems and Technology 4, 1, 2.

Zhiyuan Cheng, James Caverlee, Kyumin Lee, and Daniel Z. Sui. 2011. Exploring millions of footprints in location sharing services. In Proceedings of the 5th International AAAI Conference on Weblogs and Social Media. 81-88.

Jennifer Golbeck. 2009. Trust and nuanced profile similarity in online social networks. ACM Transactions on the Web 3, 4, 12.

Marta C. Gonzalez, Cesar A. Hidalgo, and Albert-Laszlo Barabasi. 2008. Understanding individual human mobility patterns. Nature 453, 7196, 779-782.

Tian-Ran Hu, Jie-Bo Luo, Henry Kautz, and Adam Sadilek. 2015. Home location inference from sparse and noisy data: Models and applications. In IProceedings of the 2015 IEEE International Conference on Data Mining Workshop. IEEE, Los Alamitos, CA, 1382-1387.

Chao Huang and Dong Wang. 2016a. Exploiting spatial-temporal-social constraints for localness inference using online social media. In Proceedings of the 2016 IEEE/ACM International Conference on Advances in Social Networks Analysis and Mining. IEEE, Los Alamitos, CA, 287-294.

Chao Huang and Dong Wang. 2016b. Topic-aware social sensing with arbitrary source dependency graphs. In Proceedings of the 2016 15th ACM / IEEE International Conference on Information Processing in Sensor Networks. IEEE, Los Alamitos, CA, 1-12.

Chao Huang, Dong Wang, and Nitesh Chawla. 2015. Towards time-sensitive truth discovery in social sensing applications. In Proceedings of the 2015 IEEE 12th International Conference on Mobile Ad Hoc and Sensor Systems. IEEE, Los Alamitos, CA, 154-162.

David Jurgens. 2013. That's what friends are for: Inferring location in online social media platforms based on social relationships. In Proceedings of the 7th International AAAI Conference on Weblogs and Social Media. 273-282.

Kazuki Kodama, Yuichi Iijima, Xi Guo, and Yoshiharu Ishikawa. 2009. Skyline queries based on user locations and preferences for making location-based recommendations. In Proceedings of the 2009 International Workshop on Location Based Social Networks. ACM, New York, NY, 9-16.

Cliff A. C. Lampe, Nicole Ellison, and Charles Steinfield. 2007. A familiar face (book): Profile elements as signals in an online social network. In Proceedings of the SIGCHI Conference on Human Factors in Computing Systems. ACM, New York, NY, 435-444.

Rui Li, Shengjie Wang, and Kevin Chen-Chuan Chang. 2012a. Multiple location profiling for users and relationships from social network and content. Proceedings of the VLDB Endowment 5, 11, 1603-1614. 
Rui Li, Shengjie Wang, Hongbo Deng, Rui Wang, and Kevin Chen-Chuan Chang. 2012b. Towards social user profiling: Unified and discriminative influence model for inferring home locations. In Proceedings of the 18th ACM SIGKDD International Conference on Knowledge Discovery and Data Mining. ACM, New York, NY, 1023-1031.

Chris Y. T. Ma, David K. Y. Yau, Nung Kwan Yip, and Nageswara S. V. Rao. 2013. Privacy vulnerability of published anonymous mobility traces. IEEE/ACM Transactions on Networking 21, 3, 720-733.

Jermaine Marshall, Munira Syed, and Dong Wang. 2016. Hardness-aware truth discovery in social sensing applications. In Proceedings of the 2016 International Conference on Distributed Computing in Sensor Systems. IEEE, Los Alamitos, CA, 143-152.

Jermaine Marshall and Dong Wang. 2016. Mood-sensitive truth discovery for reliable recommendation systems in social sensing. In Proceedings of the 10th ACM Conference on Recommender Systems. ACM, New York, NY, 167-174.

Jeffrey McGee, James Caverlee, and Zhiyuan Cheng. 2013. Location prediction in social media based on tie strength. In Proceedings of the 22nd ACM International Conference on Conference on Information and Knowledge Management. ACM, New York, NY, 459-468.

CUDA NVIDIA. 2008. Programming Guide.

Moon-Hee Park, Jin-Hyuk Hong, and Sung-Bae Cho. 2007. Location-based recommendation system using Bayesian users preference model in mobile devices. In Ubiquitous Intelligence and Computing. Springer, 1130-1139.

Ulrike Pfeil, Raj Arjan, and Panayiotis Zaphiris. 2009. Age differences in online social networking-a study of user profiles and the social capital divide among teenagers and older users in MySpace. Computers in Human Behavior 25, 3, 643-654.

Foster Provost, Brian Dalessandro, Rod Hook, Xiaohan Zhang, and Alan Murray. 2009. Audience selection for on-line brand advertising: Privacy-friendly social network targeting. In Proceedings of the 15th ACM SIGKDD International Conference on Knowledge Discovery and Data Mining. ACM, New York, NY, 707-716.

L. Ramaswamy, P. Deepak, R. Polavarapu, K. Gunasekera, D. Garg, K. Visweswariah, and S. Kalyanaraman. 2009. Caesar: A context-aware, social recommender system for low-end mobile devices. In Proceedings of the 10th International Conference on Mobile Data Management: Systems, Services, and Middleware. IEEE, Los Alamitos, CA, 338-347.

C. Ratti, S. Williams, D. Frenchman, and R. M. Pulselli. 2006. Mobile landscapes: Using location data from cell phones for urban analysis. Environment and Planning B: Urban Analytics and City Science 33, 5, 727.

Dong Wang, Tarek Abdelzaher, and Lance Kaplan. 2014a. Surrogate mobile sensing. IEEE Communications Magazine 52, 8, 36-41.

Dong Wang, Md Tanvir Al Amin, Tarek Abdelzaher, Dan Roth, Clare R. Voss, Lance M. Kaplan, Stephen Tratz, Jamal Laoudi, and Douglas Briesch. 2014b. Provenance-assisted classification in social networks. IEEE Journal of Selected Topics in Signal Processing 8, 4, 624-637.

Dong Wang and Chao Huang. 2015. Confidence-aware truth estimation in social sensing applications. In Proceedings of the 12th Annual IEEE International Conference on Sensing, Communication, and Networking. IEEE, Los Alamitos, CA, 336-344.

Dong Wang, Lance Kaplan, Hieu Le, and Tarek Abdelzaher. 2012. On truth discovery in social sensing: A maximum likelihood estimation approach. In Proceedings of the 11th International Conference on Information Processing in Sensor Networks. ACM, New York, NY, 233-244.

Ryen W. White, Peter Bailey, and Liwei Chen. 2009. Predicting user interests from contextual information. In Proceedings of the 32nd International ACM SIGIR Conference on Research and Development in Information Retrieval. ACM, New York, NY, 363-370.

Hongzhi Yin, Yizhou Sun, Bin Cui, Zhiting Hu, and Ling Chen. 2013. LCARS: A location-content-aware recommender system. In Proceedings of the 19th ACM SIGKDD International Conference on Knowledge Discovery and Data Mining. ACM, New York, NY, 221-229.

Jia-Dong Zhang and Chi-Yin Chow. 2015. GeoSoCa: Exploiting geographical, social and categorical correlations for point-of-interest recommendations. In Proceedings of the 38th International ACM SIGIR Conference on Research and Development in Information Retrieval. ACM, New York, NY, 443-452.

Received January 2016; revised October 2016; accepted December 2016 